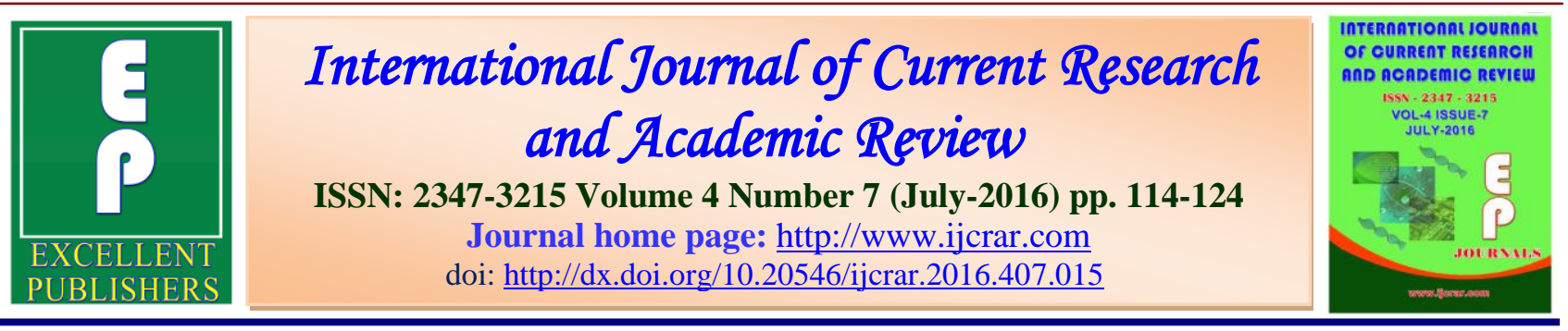

\title{
Mathematical Modeling of Content Based Image Classification Techniques for Artificial Vision
}

\author{
Rajashri Mahajan* and S.V. Patil \\ E \& TC Department, J.T. Mahajan College of Engineering, Faizpur, India \\ *Corresponding author
}

KEYWORDS

CBIR, Image

Retrieval, SVM, Image classification, image processing, pattern recognition, computer vision.

\section{A B S T R A C T}

Recent years have seen a rapid increase in the size of digital image collections. Any advance in our ability to organize unlabelled images according to their semantic content is a very useful step in managing these collections. Image is a collection of row and column that is called pixel values. Extracting best matched image from large collection of database is emerging task. Image retrieval is mainly used in image processing, pattern recognition and computer vision. CBIR technique used in many areas such as medical, academic, art, fashion, entertainment. Generally image have colour, texture, shape and size are relevant feature so extract all the relevant and irrelevant features of image. After extracting all the feature of image applies SVM i.e. supervised learning algorithm get optimal result for image classification.

\section{Introduction}

The main goal of CBIR to build new technique for extracting similar image from large collection of image i.e. database based on their content or features. Image retrieval system is used to find out similar image to query image. In CBIR, image retrieved low level feature of image like colour, shape, texture and high level feature entropy, mean, standard deviation.

Colour feature use image histogram technique, entropy gives statistical representation of image and text feature gives polite and regularity of histogram. It is most widely used technique for managing, searching, browsing and extracting visual content of image from large collection of images. These feature are stored in database for further use, when we need to find out an image, give a query image for matching, the feature of query image are extracted and matched by stored database image, so that a group of same image comes from the query image as a result.

To reduce complexity of CBIR system and to increase precision a feature reduction or feature selection algorithm is used. It uses an automatic indexing scheme, to reduce search time of retrieval system from the database. 
At searching time, user select best matched image from resultant image and then repeat the process again new matched image found. Repeat the step until an image matches to query image. Here Support Vector Machine used to find out optimal result of all the feature of image. It improves efficiency as well as accuracy of all the process of CBIR.

\section{Content Based Image Retrieval}

The block diagram shows the pertinence between a query image and resultant image. It applies feature extraction method to all the images and matches the features of query image and resultant image. An image that has best similar feature is extracted from database.

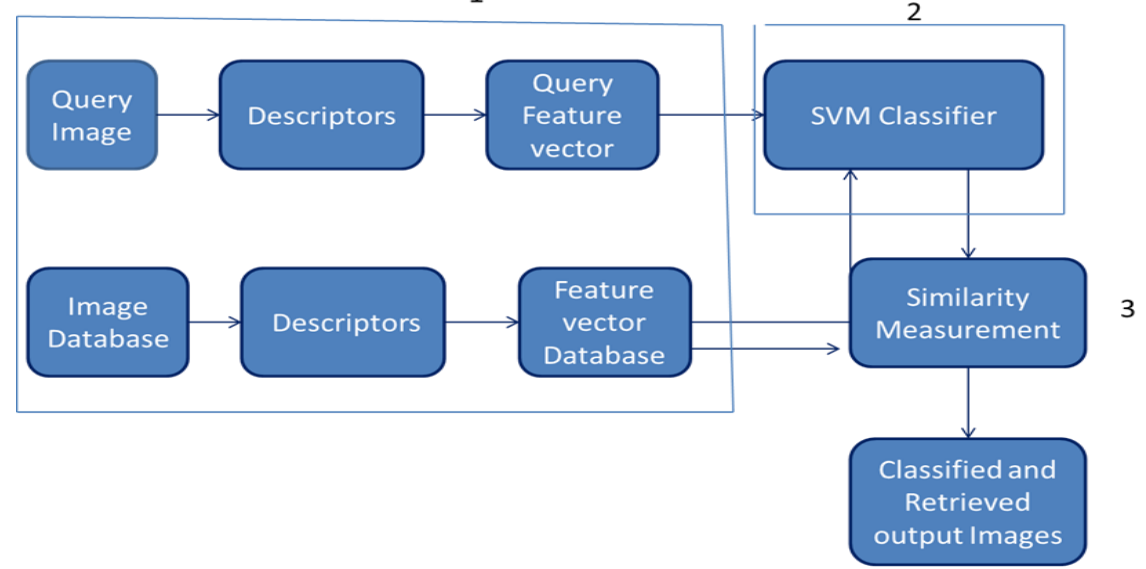

\section{Colour Feature}

Colour is one of the most important features in CBIR. It is most widely used for both human perception \& computer vision. In colour feature extraction mainly image histogram value calculated. Image histogram is a graphical representation of an image. As we know image is a collection of pixels i.e. row and column, so the image histogram shows the proportion of pixels of each colour within the image. Image histogram for each image is then stored in the database. At search time user can specify the desired proportion of each colour. Image histogram shows how many times the particular colours occur in image. The main advantage of histogram is variation in scale, rotation \&translation of image. Currently RGB i.e. Red, Green, Blue colour model is used in digital image because it is more convenient for displaying image in CRT. But it does not give good result in CBIR so we use HSV which is mostly used in CBIR system. In this colour should be matched with human expectation. In this, Hue represents different colours, saturation represents percentage of white colour and Value represents light intensity. Advantages of HSV are suitable with human perception [GuoyongDuan et al., 2011].

\section{Texture features}

Texture refers to a structural collection of pixels of an image. Many features of image can be extracted by texture features. (GLCM). He proposed some parameters for texture extraction [NidhiSingh et al., 2012].

Contrast (Moment of inertia): Image contrast can be a sharpness of image. Contrast is higher whenimage grooves are deep [GuoyongDuan et al., 2011].

Energy: It can be measure by gray distribution of image. Coarness of image depends on high energy level. 
Entropy: It is a quantity which is used to describe the amount of information of an image. If entropy level is low, then image having more black area. An image that is perfect having entropy zero [NidhiSingh et al., 2012].

Correlation: It is used to calculate the degree of similarity of the elements of image.

\section{Wavelet Features}

A wavelet is a mathematical function used to divide a given function into different frequency components. A wavelet transform is the representation of a function by wavelets, which represent scaled and translated copies of a finite length or fastdecaying oscillating waveform (known as the "mother wavelet"). Wavelet analysis represents a windowing technique with variable-sized regions.

Wavelet analysis allows the use of long time intervals where more precise low-frequency information is needed, and shorter regions where high frequency information is necessary.All wavelet transforms may be considered forms of time frequency representation for continuous-time (analog)signals.
The discrete wavelet transform (DWT) of a signal is calculated by passing it through a series of filters (high and low pass filters) and then down-sampled. At each level, the signal is decomposed into low and high frequencies, and this decomposition halves the resolution since only half the number of samples are retained to characterize the entire signal

\section{Support Vector Machine}

SVM is a supervised learning process in machine learning. The main purpose of SVM is to build optimal separating hyper planes. It accepts data and identifies patterns which are used for classification and regression analysis [Patheja et al., 2012]. It takes a set of input data and produces an inferred function called classifier (if input is discrete) or regression (if output is continuous) [Keping Wang Xiaojie Wang et $a l ., 2010]$. The main aim is to draw hyper plan as wide as possible for a good separation that means largest distance to nearest training data of pixel values [Vanitha et al., 2011]. The distance between two hyper planes is the margin of the hyper planes with respect to the sample. The purpose of SVMs is to maximize this distance .If distance of pixels to hyper plan is large than generalization error of classifier is low [Patheja et al., 2012].

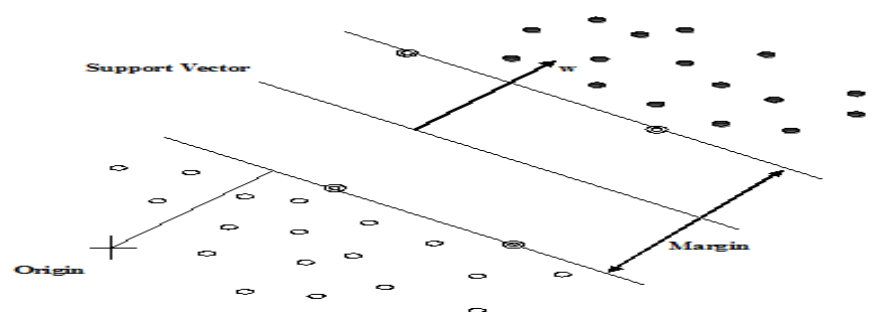

SVMs method consists of the following phases:

1.Mapping input data to high-dimensional feature space.
2. Selecting a kernel and computes the hyper planes.

3. To maximize the distance from the closest points, this is called the margin.

4. To detect the outer boundaries. 
Its performance was auspicious because it reduces prediction error and complexity at the same time.

\section{Feature Extraction}

In the proposed system there are three phases:

Pre-processing, Feature extraction, SVM classifier

Pre-processing: the main aim of preprocessing is to remove the noise and error from image. The purpose of removing noise is to get error free exact extraction of feature. If any noise remains in image then extraction gives unsatisfactory result.

Feature extraction: In this step more features of image like colour, texture, shape, mean, standard deviation, histogram value, length to width ratio, entropy are extracted.

SVM classifier: SVM is act as a classifier. Basically it is used for image classification of those features of image which are extracted from previous step.
Following figure shows the step wise working of feature extraction. When input image is ready for feature extraction first it goes through pre-processing phase than goes to next phase here feature extraction is performed.

\section{Flow of Content Based Image Categorization System Flow of Content Based Image Categorization System Using Color Feature}

In this work the images are categorized based on color feature. Main steps involved in this workare extraction of features, training of SVM and classification.

\section{Flow of Content Based Image Categorization System Using Texture Feature}

In this system categorization is carried out using texture which is an important low level feature. The features like energy, entropy, homogeneity, correlation are calculated.

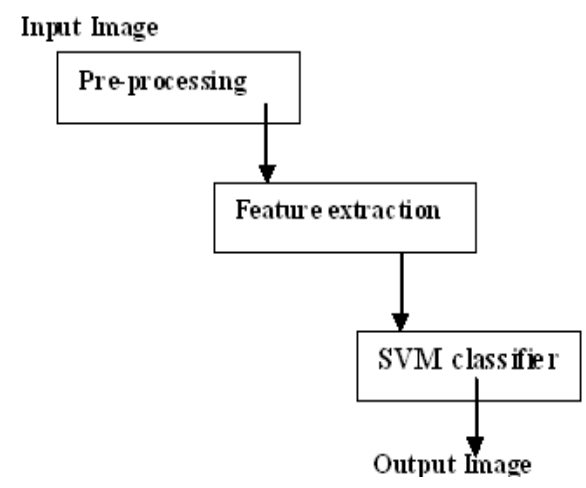

Block diagram of Feature extraction system 


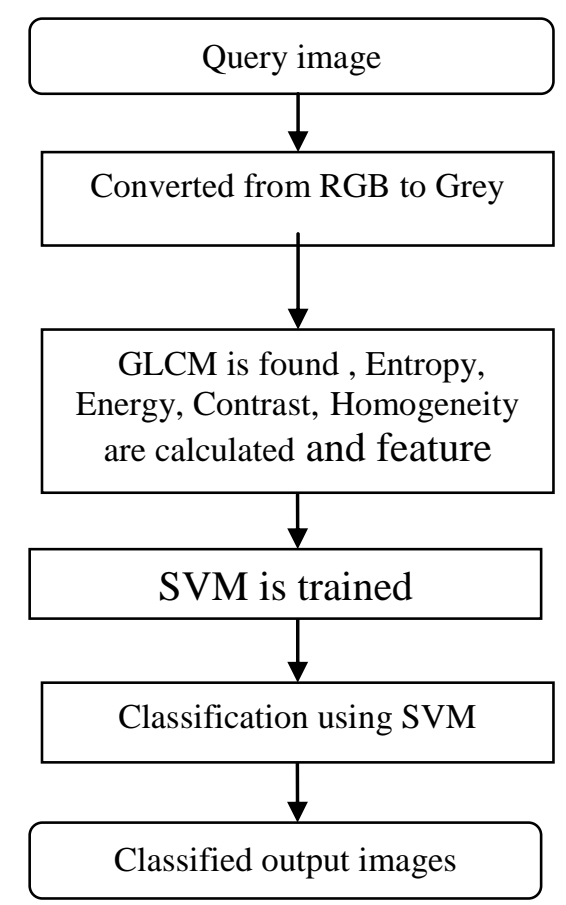

\section{Flow of Content Based Image Categorization System Using Wavelet Feature}

In this section the image is first converted from RGB to Grey.
Coiflet wavelet transform is then allpied to grey image. The coefficients are used to create feature vector.

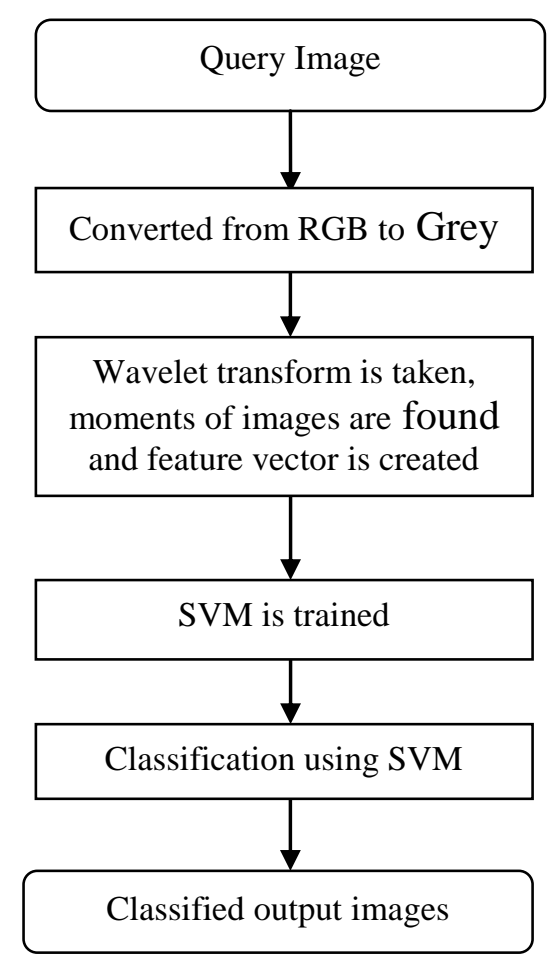


Int.J.Curr.Res.Aca.Rev.2016; 4(7): 114-124

Flow of Content Based Image features such as color histogram, color Categorization System Using Color $+\quad$ moments, auto correlogram are found. Texture Feature Similarly texture features are extracted from image by using GLCM .

In this system combination of color and texture feature is used. First the color

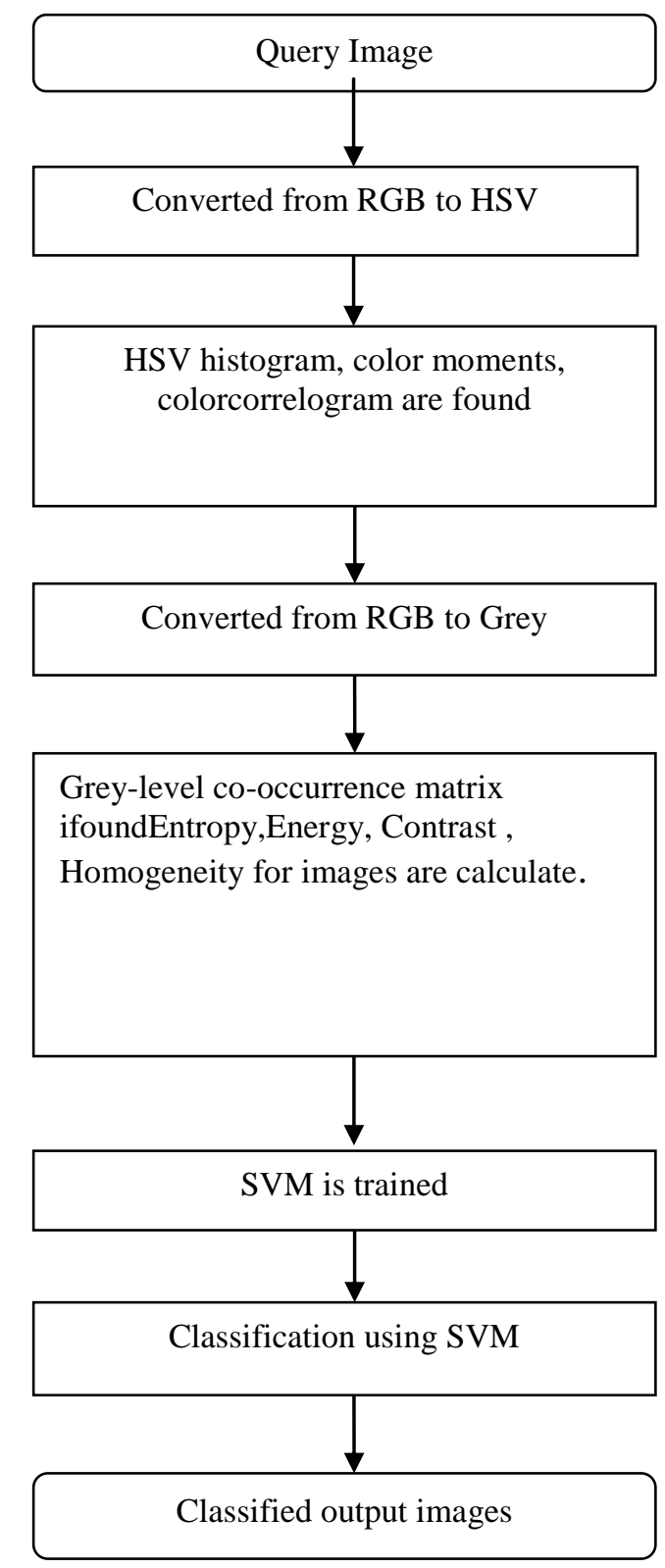

Flow of Content Based Image Categorization System Using Color + Wavelet Feature

In this system combination of color and wavelet feature is used.

First the color and wavelet features are extracted from image. These features are used to create feature vectors. 


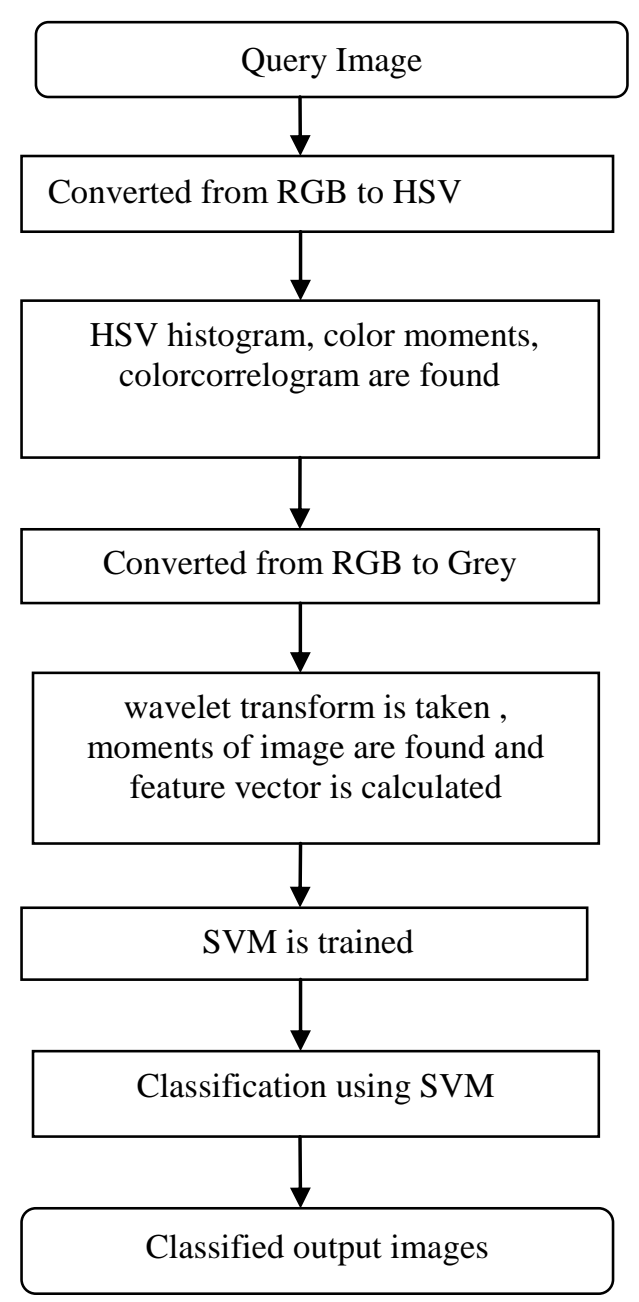

\section{Results and Discussion}

Algorithms for categorization based on different image features are implemented in matlab on 500 images from SIMPLicity database in .jpg format and size $384 \times 256$ or $256 \times 384$. The database used contains five different categories, 100 cityscopes, 100 Interior designs, 100 Big trucks, 100 Apples, 100 birds. Here three well known parameters are used to evaluate the performance of the image classification algorithm accuracy, precision and recall. positives (i.e. the number of items correctly labeled as belonging to the positive class) divided by the total number of elements labeled as belonging to the positive class.

$$
\text { Precision }=\frac{t_{p}}{t_{p}+f_{p}}
$$

Recall :Recall in this context is defined as the number of true positives divided by the total number of elements that actually belong to the positive class

Accuracy $=\frac{\text { Sum of correctly classified pixels }}{\text { Total no. of pixels }}=\frac{\text { Sum of diagonal elereegat }}{\text { Total no. of pixels }}=\frac{t_{p}}{t_{p}+f_{n}}$

Precision: In a classification task, the Where, $t_{p}$ : true positive predictions, $f_{p}$ : precision for a class is the number of true false positive predictions, $\mathrm{f}_{\mathrm{n}}$ : false negative predictions 
Int.J.Curr.Res.Aca.Rev.2016; 4(7): 114-124

Table.1 Performance analysis of image categorization on the basis of Precision and Recall for color feature

\begin{tabular}{|c|c|c|c|c|}
\hline \multirow{2}{*}{ Class } & \multicolumn{4}{|c|}{ Using color } \\
\cline { 2 - 5 } & \multicolumn{2}{|c|}{ Using L1 } & \multicolumn{2}{c|}{ Using L2 } \\
\cline { 2 - 5 } & Precision & Recall & Precision & Recall \\
\hline Cityscopes & 0.69885 & 0.67172 & 0.68263 & 0.7504 \\
\hline Interiordesign & 0.73079 & 0.70063 & 0.70428 & 0.72803 \\
\hline Big trucks & 0.76344 & 0.69274 & 0.70428 & 0.72803 \\
\hline Apples & 0.65415 & 0.7333 & 0.6938 & 0.73279 \\
\hline Birds & 0.73239 & 0.70582 & 0.70020 & 0.72319 \\
\hline
\end{tabular}

Table.2 Performance analysis of image categorization on the basis of Precision and Recall for Texture feature

\begin{tabular}{|c|c|c|c|c|}
\hline \multirow{2}{*}{ Class } & \multicolumn{3}{|c|}{ Using Texture } \\
\cline { 2 - 5 } & \multicolumn{2}{|c|}{ Using L1 } & \multicolumn{2}{c|}{ Using L2 } \\
\cline { 2 - 5 } & Precision & Recall & Precision & Recall \\
\hline Cityscopes & 0.97538 & 0.95340 & 0.9689 & 0.94875 \\
\hline Interiordesign & 0.98487 & 0.95427 & 0.97538 & 0.95340 \\
\hline Big trucks & 0.98529 & 0.975 & 0.995 & 0.96459 \\
\hline Apples & 0.97979 & 0.94479 & 0.98409 & 0.956 \\
\hline Birds & 0.95599 & 0.98944 & 0.96665 & 0.99479 \\
\hline
\end{tabular}

Table.3 Performance analysis of image categorization on the basis of Precision and Recall for Wavelet feature

\begin{tabular}{|c|c|c|c|c|}
\hline \multirow{2}{*}{ Class } & \multicolumn{4}{|c|}{ Using Wavelet } \\
\cline { 2 - 5 } & \multicolumn{2}{|c|}{ Using L1 } & \multicolumn{2}{c|}{ Using L2 } \\
\cline { 2 - 5 } & Precision & Recall & Precision & Recall \\
\hline Cityscopes & 0.98584 & 0.96904 & 0.85053 & 0.86743 \\
\hline Interiordesign & 0.82706 & 0.84508 & 0.833 & 0.85 \\
\hline Big trucks & 0.78423 & 0.88338 & 0.7522 & 0.84784 \\
\hline Apples & 0.82641 & 0.8439 & 0.83367 & 0.84611 \\
\hline Birds & 0.78129 & 0.92439 & 0.77154 & 0.88333 \\
\hline
\end{tabular}

Table.4 Performance analysis of image categorization on the basis of Precision and Recall for Color + Texture

\begin{tabular}{|c|c|c|c|c|}
\hline \multirow{2}{*}{ Class } & \multicolumn{4}{|c|}{ Using Color + Texture } \\
\cline { 2 - 5 } & \multicolumn{3}{|c|}{ Using L1 } & \multicolumn{2}{c|}{ Using L2 } \\
\cline { 2 - 5 } & Precision & Recall & Precision & Recall \\
\hline Cityscopes & 0.84137 & 0.91195 & 0.85409 & 0.87983 \\
\hline Interior design & 0.85634 & 0.91744 & 0.85833 & 0.78510 \\
\hline Big trucks & 0.86837 & 0.91723 & 0.86075 & 0.82231 \\
\hline Apples & 0.88704 & 0.85745 & 0.88419 & 0.86590 \\
\hline Birds & 0.91242 & 0.89692 & 0.85303 & 0.96201 \\
\hline
\end{tabular}


Int.J.Curr.Res.Aca.Rev.2016; 4(7): 114-124

Table.5 Performance analysis of image categorization on the basis of Precision and Recall for Color + Wavelet

\begin{tabular}{|c|c|c|c|c|}
\hline \multirow{2}{*}{ Class } & \multicolumn{4}{|c|}{ Using Color + Wavelet } \\
\cline { 2 - 5 } & \multicolumn{2}{|c|}{ Using L1 } & \multicolumn{2}{c|}{ Using L2 } \\
\cline { 2 - 5 } & Precision & Recall & Precision & Recall \\
\hline Cityscopes & 0.83805 & 0.81473 & 0.84523 & 0.7919 \\
\hline Interiordesin & 0.84500 & 0.87165 & 0.83620 & 0.90473 \\
\hline Big trucks & 0.85056 & 0.85243 & 0.85049 & 0.83229 \\
\hline Apples & 0.86628 & 0.85978 & 0.82130 & 0.85380 \\
\hline Birds & 0.82231 & 0.86724 & 0.83472 & 0.88758 \\
\hline
\end{tabular}

Table.6 Accuracy using (Manhattan Distance) L1

\begin{tabular}{|c|c|c|c|c|c|}
\hline \multirow{2}{*}{ Class } & \multicolumn{5}{|c|}{ ACCURACY in (\%) using L1 } \\
\cline { 2 - 6 } & $\begin{array}{c}\text { Using } \\
\text { Texture }\end{array}$ & $\begin{array}{c}\text { Using } \\
\text { Color }\end{array}$ & $\begin{array}{c}\text { Using } \\
\text { Wavelet }\end{array}$ & $\begin{array}{c}\text { Using Color } \\
\text { + Texture }\end{array}$ & $\begin{array}{c}\text { Using Color } \\
\text { + Wavelet }\end{array}$ \\
\hline Cityscopes & 96.40 & 59.25 & 79.20 & 55.20 & 72.80 \\
\hline Interior design & 94.00 & 72.80 & 78.90 & 95.20 & 71.20 \\
\hline Big trucks & 96.40 & 54.80 & 74.80 & 81.20 & 72.80 \\
\hline Apples & 96.00 & 80.80 & 74.00 & 69.60 & 63.60 \\
\hline Birds & 94.00 & 56.00 & 79.20 & 80.80 & 74.00 \\
\hline
\end{tabular}

Table.7 Accuracy using (Euclidean Distance) L2

\begin{tabular}{|c|c|c|c|c|c|}
\hline \multirow{2}{*}{ Class } & \multicolumn{5}{|c|}{ ACCURACY (in \%) using L2 } \\
\cline { 2 - 6 } & $\begin{array}{l}\text { Using } \\
\text { Texture } \\
\text { Color }\end{array}$ & $\begin{array}{l}\text { Using } \\
\text { Using Wavelet }\end{array}$ & $\begin{array}{l}\text { Using } \\
\text { Color }+ \\
\text { Texture }\end{array}$ & $\begin{array}{l}\text { Using Color + } \\
\text { Wavelet }\end{array}$ \\
\hline Cityscopes & 95.20 & 52.80 & 76.00 & 83.60 & 72.00 \\
\hline Interiordesign & 93.60 & 58.60 & 78.12 & 77.20 & 74.80 \\
\hline Big trucks & 92.67 & 95.20 & 72.80 & 57.60 & 77.60 \\
\hline Apples & 77.20 & 55.20 & 77.20 & 78.00 & 83.20 \\
\hline Birds & 79.20 & 56.00 & 79.20 & 80.00 & 74.00 \\
\hline
\end{tabular}


Table.8 Output Images For Cityscopes Class

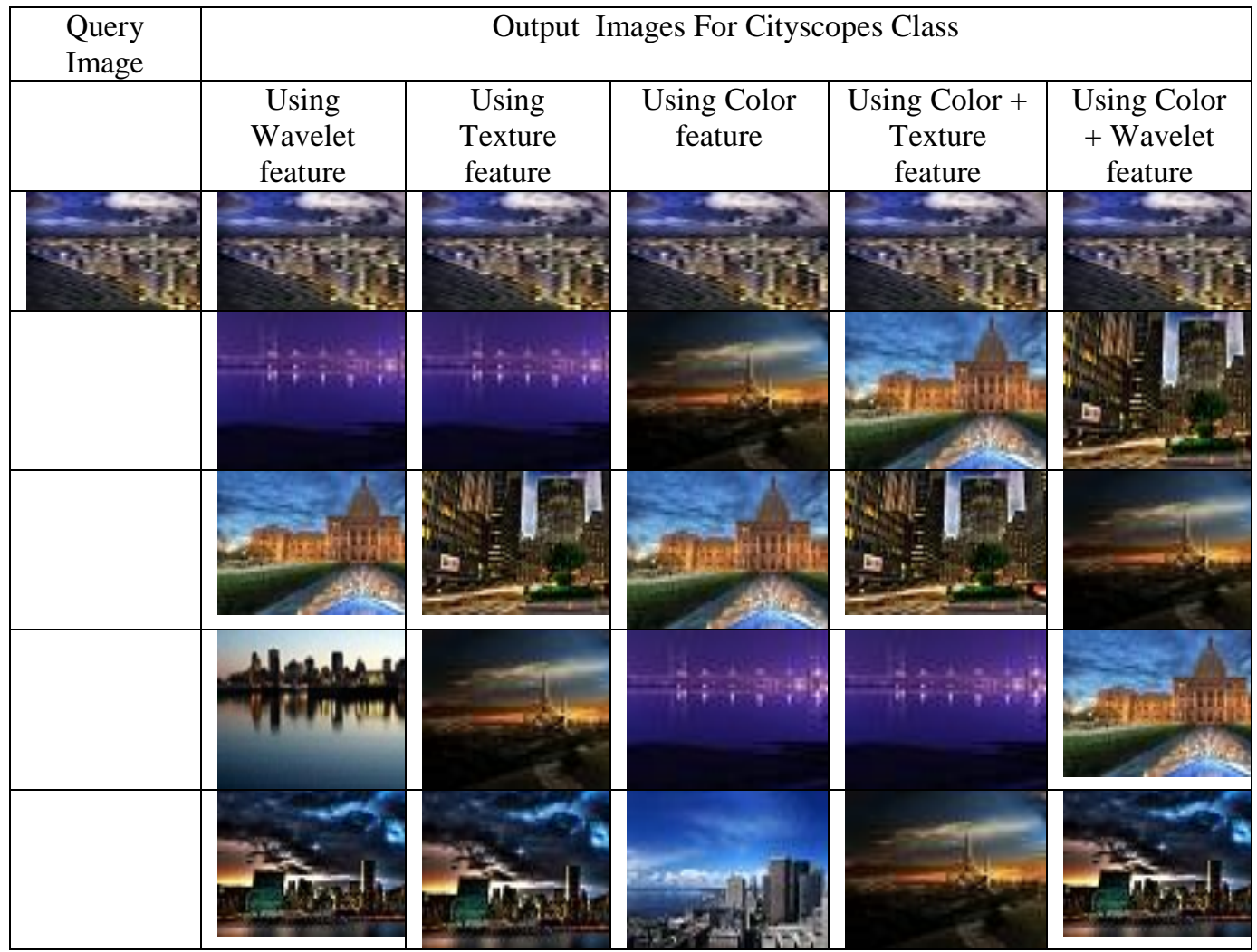

Result of Categorization Using Color Feature

Experiment is carried out on 500 images of SIMPLIcity database. Here categorization is based on color feature and its performance is evaluated by calculating accuracy, precision and recall.

\section{Result of Categorization Using Texture Feature}

This method utilizes texture feature for the categorization process. The parameters accuracy, precision and recall are calculated for both Manhattan distance(L1) and Euclidean distance (L2)

\section{Result of Categorization Using Wavelet Feature}

In this method coiflet wavelet is used for categorization process. The parameters accuracy, precision and recall for Manhattan distance (L1) and Euclidean distance

Result of Categorization Using Color + Texture Feature

In this section combination of color and texture is used for categorization. Experiment is carried out on images taken from SIMPLIcity database and results are enlisted in the table 4.4

\section{Result of Categorization Using Color + Wavelet Feature}

The categorization is performed using combination of color and wavelet. The corresponding values of accuracy, precision and recall for Manhattan distance and Euclidean distance are enlisted in table 4.5

\section{Accuracy Using L1}

The accuracy values for the implemented methods are enlisted in following table 


\section{Accuracy Using L2}

The accuracy values for the implemented methods are enlisted in following table

\section{Output Images}

Output images retrieved for each class after categorization procedure. Here select a fig.no.4 from database of cityscopes as a query image and returned images are found by their features like color feature, texture feature, wavelet feature, color+texture feature, color+wavelet feature. This image is from Cityscopes class.

\section{Conclusion}

We have extracted features such as color, texture, and histogram value of an image using feature extraction method. In this proposed work we have used SVM as a classifier for classification of various categories of image such as City scopes, Interior design, Bigtrucks, Apples, Birds. Accuracy of the classifier using texture feature seems to be better as compared to that using color and wavelet. Highest value of accuracy is $96.40 \%$ for Big trucks class using texture while it is $79.20 \%, 72.80 \%$ for Interior designs classes using wavelet and color respectively. The accuracy of color + texture combination is high for Birds class $80.00 \%$ and it is high for City scopes class using combination of color + wavelet $72.80 \%$. Also the accuracy value is better for combination of color and texture as compared to color feature only. The precision and recall values for the color feature are again poor as compared to texture, wavelet, combination of color and wavelet, color and texture.

\section{References}

Keping Wang Xiaojie Wang, YixinZhong. 2010. "A Weighted Feature Support Vector Machines Method forSemantic Image Classification", International Conference on Measuring Technology and Mechatronics Automation (IEEE).

Ing, H., Ney, Thomas Deselaers Matrikel nummer. 2003. "Features for Image Retrieval".

Guoyong Duan, Jing Yang, Yilong Yang. 2011. "Content-Based Image Retrieval Research", International Conference on Physics Science and Technology.

Nidhi Singh, Kanchan Singh, Ashok, K., Sinha. "A Novel Approach for Content Based Image Retrieval", Procedia Technology 4, C3IT- 2012.

Fazal-E-Malik and Baharum Bin Baharudin. 2011. "Mean And Standard Deviation Features Of Color Histogram using Laplacian Filter For Content-Based Image Retrieval", J. Theoretical and Appl. Information Technol., Vol. 34 No.1.

Kekre, H.B., Kavita Patil. 2009. " Standard Deviation of Mean and Variance of Rows and Columns of Images for CBIR", World Academy of Science, Engineering and Technology 272009.

Vanitha. L. and Venmathi, A.R, 2011. Sriperumbadur, "Classification of medical images using Support Vector Machine", International conference on information and network technology.

Patheja, P.S., Waoo Akhilesh, A., Maurya Jay Prakash. 2011. "An Enhanced Approch for Content Based Image Retrieval", Res. J. Recent Sci., ISSN 2277 - 2502 Vol. 1(ISC-2011), 2012.

\section{How to cite this article:}

Rajashri Mahajan and S.V. Patil. 2016. Mathematical Modeling of Content Based Image Classification Techniques for Artificial Vision. Int.J.Curr.Res.Aca.Rev.4(7): 114-124. doi: http://dx.doi.org/10.20546/ijcrar.2016.407.015 